\title{
DYNAMIC CURATIVE MECHANISM FOR GEOGRAPHIC ROUTING IN WIRELESS MULTIMEDIA SENSOR NETWORKS
}

\author{
Mohamed Nacer Bouatit, Selma Boumerdassi \\ Centre d'Etude et de Recherche en Informatique et Communications / \\ Conservatoire National des Arts et Métiers - Paris
}

\begin{abstract}
Maintaining network stability and extending network lifetime to cope with breaking links and topology changes remain nowadays a unsolved issues in Wireless Multimedia Sensor Networks (WMSNs), which aim to ensure flow delivery while guaranteeing QoS requirements, particularly, during data transmission phase. Therefore, in this paper, we jointly consider multipath transmission, load balancing and fault tolerance, to enhance the reliability of transmitted data. We propose a Dynamic Curative Mechanism for Geographic Routing in WMSNs. Theoricals results and those obtained from simulation study demonstrate the validity and efficiency of our proposed mechanism, and indicate that it is highly advised for multimedia transmission and network stability.
\end{abstract}

\section{KEYWORDS}

Wireless Multimedia Sensor Networks, Fault-Tolerance, Geographic Multipath Transmission, Load Balancing.

\section{INTRODUCTION}

Latest advances in wireless networks and MEMS technologies (Micro-Electro-MechanicalSystems) and embedded systems have led to the creation of Wireless Sensor Networks (WSNs). With a small size and low cost, wireless sensors can be deployed in hundreds over a wide area, to take scalar measurements of physical phenomena, such as temperature, pressure, humidity or the location of objects. In general, these applications are tolerant of delay and do not require a high bandwidth.

The vulgarization of miniaturized technological devices, such as cameras and microphones, has favoured the development of Wireless Multimedia Sensors Networks (WMSNs). Equipped with an on-board camera, these new sensors provide a better description of the observed phenomena by collecting, in addition to scalar data, multimedia content (video and audio streams). On the other hand, they pose new challenges in terms of ensuring QoS parameters, such as bandwidth, data delivery rate, and end-to-end transmission delay, as well as the limited energy of sensor nodes, which consequently reduces network lifetime and causes instability during data transmission phase. This last one is usually subject to ruptures due the unreliability of wireless links and also to topology changes especially in case of nodes' mobility. 
In addition to inherited constraints of classical sensor networks (energy, storage and computing), a common problem in such networks is information error, loss caused by components failure, wireless transmission error and external interference [1].

Fault tolerance is considered as an importance domain among various fields of research in wireless sensor networks, due to their constraints such as energy limitations, environment and deployment, where redeployment prohibitive cost, presents a handicap for reorganization network in case of failures of one or more of its components.

Various existing solutions proposed for fault tolerance in WSNs suffer from a poor trade-off between the scalability of the system and the level of tolerance offered by produced topology [2] and they do not cover the issues related to broken communication links and network stability by conserving operational paths during data transmission phase.

In this paper, we propose a Dynamic Curative Mechanism for geographic routing, in order to ensure this trade-off and to meet multimedia transmission constraints:

Our proposed Dynamic Curative Mechanism for geographic routing supports two features:

1. Dynamic load balancing strategy: which dynamically distributes the flow between operational paths without interrupting transmission, in order to prevent packets' early loss and provides better load balancing;

2. Local repair strategy: repair locally broken links by bypassing dynamic holes formed during transmission, due to energy depletion, physical destruction or topology changes.

The remainder of this paper is organized as follows. Section II, discusses fault tolerance procedure's and gives a classification of existing solutions. Section III, presents network model. Proposed DCM is described in section IV. Results of extensive simulations and experimentation are shown in section IV. Finally, section V, concludes the paper.

\section{RELATED WORK}

Energy limitation and hostile environment in which sensor nodes are deployed, as well as the unreliability of radio communications and loss of wireless connections due to the depletion of sensor node's battery or its physical destruction are factors that increase the risk of breakdowns and make the network vulnerable.

Since physical access to these nodes is often impossible given the intended applications, such as monitoring forest fires in which sensors are scattered by air, or monitoring of urban infrastructure like bridges where sensors are incorporated in the structure. Failures and malfunctions of sensor node, produce data loss and generate topologies changes, which consequently impact network connectivity and reduce its lifetime.

The design of fault-tolerance procedure depends on the architecture and functionality of the system. Nevertheless, some steps are common to most systems [3]:

- Error detection: allows to recognize that an unexpected event occurred. Techniques used are classified into two categories, the offline detection that runs when the system is inactive while the online detection enables the identification of real-time fault and is performed during system activity. 
- Damage containment: determines failure impact limits on the system to prevent the spread to other regions.

- Error recovery: two techniques are used, in backward recovery, the system state is restored to an earlier state that is error-free, while in forward recovery, the goal is to reach a consistent state, which is error free.

- Fault treatment: after isolation of failed component, this phase repairs it according to the type of failure.

Fault-tolerant routing algorithms can be divided into two main classes depending on treatment phase: preventive algorithms use fault-tolerant techniques to delay or avoid any errors, while curative algorithms, do not trigger implemented mechanism only when failure is detected.

One can also see fault tolerance from an architectural point of view, which deals different types of component management, namely:

\subsection{Battery management}

Considered as preventive, it aims to set a uniform distribution of energy dissipation between sensors to better manage energy consumption and increase network lifetime. The Duty-Cycling technique is used to determine the percentage of activity of a node that periodically sleeps to conserve energy. In McTPGF [4], which is an extension of TPGF [5], sleeping-delay is taken into account in routing decision. Its weakness lies in the fact that authors use a single path in their studies and results proved that McTPGF reduces end-to-end delay with the cost of adding few hop counts compared with usual TPGF.

\subsection{Flow management}

This category includes data transfer management:

- Multipath routing : uses a preventive algorithm to establish multiple paths from the source to the sink. This ensures the presence of more reliable paths for transmission and offers rapid recovery of transfer in case of failures. In [6], a fault-tolerant routing protocol, that modifies conventional DSR [7] protocol, is proposed. It tries to find two routing paths from the source to the destination. Its disadvantage is the use of a single route for transmission, which causes an overload at nodes constituting this path. Additionally, in case of failure, protocol uses secondary route and therefore all packets that have borrowed the faulty path are lost.

- Route recovery: curative technique that creates an alternative new path to ensure the retransmission of data. In [8], to achieve reliability and fault tolerance, proposed protocol continuously updates routes status, while transmitting data along paths simultaneously, it uses a subsequent reliable path, if some unpredicted fault happens in the path. Its drawback is the flooding of network by updating requests and control messages, additionally, the overload of nodes involved in the transmission, which consequently deplets their residual energy.

- Channel allocation: implemented at the MAC layer, this solution performs allocation of transmission channel, in order to reduce interferences between neighboring nodes and avoids packet collisions during transfer. In [9], Channel Utilization and Delay Aware Routing protocol (CUDAR) is proposed that satisfy QoS parameters (throughput, delay and jitter) and reduce energy consumption by using adaptive channel utilization module in MAC layer.

- Mobility: this approach allows to choose a number of mobile nodes, with superior capacity (energy and calculation), which are responsible for collecting data by moving between network sensors. This allows a saving in energy consumption by reducing hops number of transmitted packets. In [10], a pragmatic approach to area coverage in hybrid WSNs is proposed to enhance and maintain the area coverage by moving mobile sensor nodes in the uncovered area. Its main drawback is that only the sink launchs the hole detection and recovery process. 


\subsection{Data management}

Solutions in this category offer better data management and processing. Two major subcategories are derived :

- Aggregation: considered as preventive approach, performs processing on the raw data collected from the environment and combines captured data from multiple nodes to reduce the amount of data transmitted across the network and thereby increases its lifetime. In [1], a combination of trust mechanism, data aggregation, and fault tolerance is proposed, to enhance data trustworthiness in WMSNs. It incurs high routing and computational overhead while exchanging trust information. This exchange of information leads to false reporting attack where a malicious node may propagate false information to decrease trust rating for well reputed node [11].

- Clustering: preventive technique (sometimes considered as curative approach) treats structure of sensor networks. It allows to form a virtual backbone for better use of resources such as bandwidth and energy. A cluster-based transmission mechanism with dynamic changes in the path has been proposed in [12]. However, its limitation is that authors assume a secure communication channel and they have not taken into account malicious attacks against trust models [13].

In this article, we focused on a curative technique that uses an optimistic approach, we have also combined two techniques of flow management: multipath routing and route recovery, in order to guarantee QoS and maintain network stability.

\section{NETWORK MODEL}

We consider a flat network architecture and the wireless sensor network is composed of $\mathrm{N}$ sensors, deployed in a static deterministic manner, each sensor node being aware of its geographic location and its 1-hop neighbor nodes' geographic locations. We assume that only source nodes defined for area of interest know the Base Station (BS) location and all other sensor nodes know BS by receiving packets from source nodes. All nodes have the same transmission range and are homogeneous and are endowed with identical physical capabilities (detection and communication). Only bidirectional links are used to build paths. Each sensor node may be in one of the following states:

- Valid: ready to build a path;

- Active: already used in a path (locked for specific path);

- Blocked: no valid next-hop except its predecessor;

- Failed: broken, damaged or exhausted battery.

Each path is composed of a finite set of links, each node can belong to only one path, except source nodes and sink node. In GMFT, all generated routing paths are node-disjoint routing paths.

Let $N=\backslash\left\{n \_1, . ., n \_m \backslash\right\}$ be the finite set of nodes, $L=\backslash\left\{1 \_1, . ., 1 \_k \mid\right\}$ be the finite set of links, and $\mathrm{P}=\backslash\left\{\mathrm{p}_{-} 1, \ldots, \mathrm{p} \_\mathrm{n} \backslash\right\}$ be the finite set of paths. MaxPathNum is the maximum path number used by source node for each flow transmission.

Static holes are the subset of failed nodes before transmission or blocked nodes, while dynamic holes are the subset of active nodes or failed nodes during data transmission phase, due to the depletion of their energy or their physical destruction. 


\section{DYNAMIC CURATIVE MECHANISM}

\subsection{Description}

The design of our DCM is based on the different characteristics related to multimedia transmission in WMSNs and is also exposed to three following sub-problems:

- Maintain network stability when communication links break;

- Ensure load balancing, to extend network lifespan;

- Bypass dynamic holes formed during data transmission phase, to avoid packets' early loss.

Our main goal is to ensure reliability of transmitted data, extend network lifetime, satisfy QoS requirements by tolerating potential faults that may occur such as, energy depletion, hardware failure, communication link errors and interferences, in order to keep the system stable and without interruption, in case of failure of some of its components.

Based on some limits of offline geographic routing protocols, where the discovery of paths is performed before sending data. Thus, constructed paths may not reflect network reality at transmission time, especially in case of mobility or breakdown of nodes. Any disturbance impacts directly the quality of multimedia streams at the receiver. Furthermore, as multimedia data transmission is generally characterized by a long duration and a large size, sensor nodes are highly likely to fail due to the depletion of their energy. Therefore, we introduced our dynamic curative mechanism based on fault detection, dynamic load balancing and local repair, to conserve network stability during data transmission phase and solve the issue related to reliability of transmitted data, hence allowing to cope with topology changes.

\subsection{Operation}

In case of failure, the curative mechanism is used when a node notices that its successor is missing by not receiving successive acknowledgments and reacts by applying:

- Dynamic load balancing strategy: it sends a blocking message to source node, to prevent packet loss and increase delivery rate. Upon receiving a blocking message, source node blocks immediately the faulty path and distributes the flow on the remaining operational paths, to ensure continuity of transmitted data.

- Local repair strategy: It initiates exploration phase similar to the one previously described. Nevertheless, it stops this action after meeting a junction node belonging to the initial broken path, and which distance from the collector is closer than failed node distance. This allows a local reparation without having to rebuild a new path from the source to the collector. When the link is established, all nodes that are no longer part of the new path are released. 


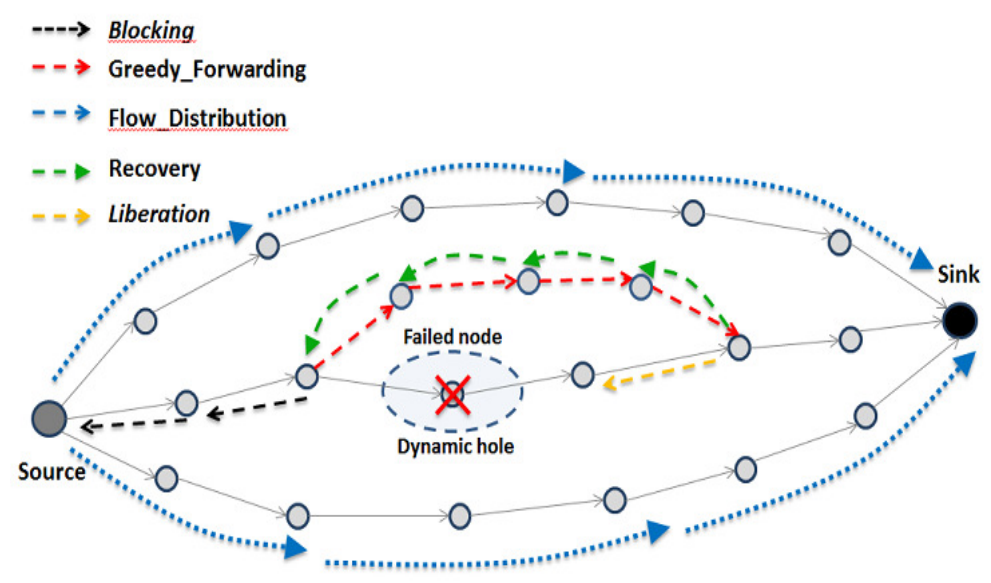

Figure 1. Dynamic curative mechanism

\subsection{Theoretical modelling}

To the best of our knowledge, all geographic routing protocols endowed with fault tolerance mechanism or not, do not support dynamic holes involving one or more nodes caused by broken links during data transmission phase.

If node $n_{i} \in N, n_{i} \in p_{j}$ with $p_{j} \in P$, fails at time $t_{n i}$, all packets which used this faulty path after $t_{n i}$ are lost. We define the Number of Lost Packets (NLP) as follows:

$$
N L P_{n_{i}}=\frac{N S P}{N_{\text {path }}}\left(1-\frac{t_{n_{i}}}{T_{S}}\right)+\beta
$$

NSP: Number of sent packets.

$\mathrm{N}_{\text {path }}$ : Number of paths.

$\mathrm{t}_{\mathrm{n}}$ : Time when node $\mathrm{n}_{\mathrm{i}}$ fails.

$\mathrm{T}_{\mathrm{S}}$ : Total simulation time.

$\beta$ : Number of lost packets due to transmission errors.

The first argument of formula (1) defines the packet loss due to path breaks, while the second argument is related to transmission errors.

In general, we obtain, with $\mathrm{N}_{\mathrm{FN}}$, Number of failed nodes during transmission phase

$$
N L P_{\left(n_{1}, \ldots, n_{F N}\right)}=\frac{N S P}{N_{\text {path }}}\left(N_{F N}-\frac{\sum_{i=1}^{F N} t_{n_{i}}}{T_{S}}\right)+\beta
$$

In our design, a node realizes the lack of its successor by not receiving successive acknowledgments after $i_{n}$ iterations number, the NLP is independent of failure time and paths containing failed nodes. This can be modelled this way:

$$
N L P=i_{n} N_{F N}+\alpha
$$

$\mathrm{i}_{\mathrm{n}}$ : Iterations number required to trigger curative mechanism;

$\alpha$ : Number of lost packets due to transmission errors. 


\section{EVALUATION}

In order to demonstrate the strength of our proposed solution, we implemented our DCM on our previous protocol [14] and conducted several simulation, based on TinyOS [15] platform which conception defers from other OSs and relies on low-energy consumption operations. We also implemented both TPGF and AGEM [16] protocols, which are geographic routing protocols cited in several recent papers, according to their advantages. TPGF is effective in optimal path discovery, while AGEM is efficient in energy, also their common strengths, the bypassing of static holes during paths construction and flow management by multipath transmission.

\begin{tabular}{|c|c|}
\hline Parameter & Value \\
\hline Network size & $500 \mathrm{~m} \mathrm{x} \mathrm{250m}$ \\
Number of sensors nodes & 253 \\
Bandwidth & $250 \mathrm{Kbits} / \mathrm{s}$ \\
Transmission range & $25 \mathrm{~m}$ \\
Packet size & $128 \mathrm{Bytes}$ \\
Data size & $3.34 \mathrm{Mb}$ \\
Number of paths & 5 \\
\hline
\end{tabular}

Table 1. Main configuration parameters

\subsection{Theoretical results}

\begin{tabular}{|c|c|c|c|c|c|}
\hline & \multicolumn{5}{|c|}{ Number of failed nodes } \\
\hline Protocols & 1 & 2 & 3 & 4 & 5 \\
\hline TPGF/AGEM & 2990 & 5981 & 8971 & 11962 & 14953 \\
\hline With DCM & $\mathbf{5}$ & $\mathbf{1 0}$ & $\mathbf{1 5}$ & $\mathbf{2 0}$ & $\mathbf{2 5}$ \\
\hline
\end{tabular}

Table 2. Theoretical number of lost packets

Table 2. shows the NLP for a total of 30000 sent packets and we assume that the time of failure of each node is the same: $\mathrm{t}_{\mathrm{n} 1}=\mathrm{t}_{\mathrm{n} 2}=\mathrm{t}_{\mathrm{n} 3}=\mathrm{t}_{\mathrm{n} 4}=\mathrm{t}_{\mathrm{n} 5}=\mathrm{Ts} / 2$.

Our dynamic curative mechanism allows a better reliability as compared with other existing solution in WMSN. Therefore, it allows to minimise the NLP in case of path breaks during data transmission phase, which had never been solved in previous works.

\subsection{Simulation results}

\subsubsection{Delivery Ratio (DR)}

Is the ratio, for a given period of time, between the Number of Received Packets (NRP) and total Number of Sent Packets (NSP), it reflects the reliability of the protocol during packets' transfer from source node to destination.

$$
D R=\frac{N R P}{N S P}=\frac{N S P-N L P}{N S P}
$$

Having an optimal DR heavily relies on reducing the NLP. Figure.2(a) shows that the success rate of TPGF and AGEM registered a significant deterioration as compared to DCM and decreases from $991 \%$ to $(481 \%, 811 \%)$ respectively for TPGF and AGEM, when five nodes belonging to different paths failed, and this is due to the non-mantainance of broken paths. One can note that 
larger the dynamic hole (several failed nodes), the smaller the delivery rate. This confirms formulas (1 and 2) mentioned above and the effectiveness of our curative mechanism.

\section{Delivery ratio}

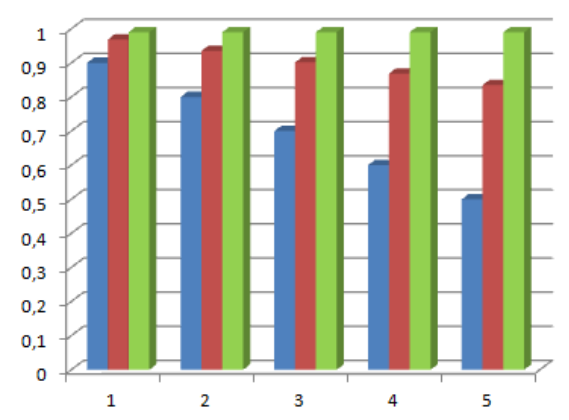

(a) Number of failed nodes

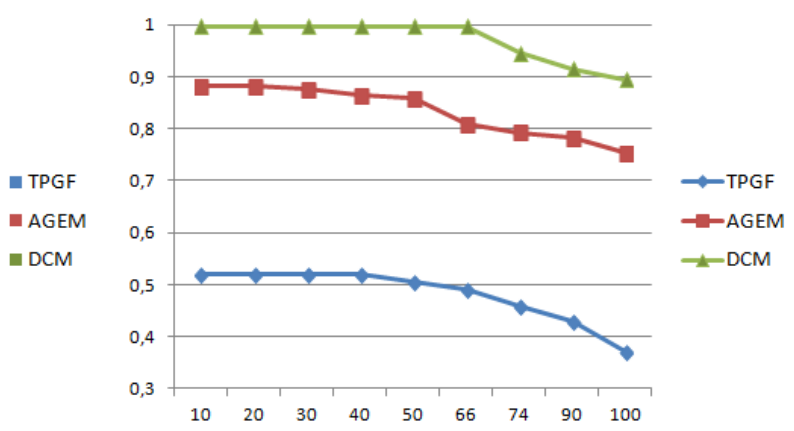

(b) Sending rate $(\mathrm{P} / \mathrm{S})$

Figure 2. Impact of number of failed nodes and sending rate on delivery ratio

\subsubsection{Receiving Rate $(\mathrm{RR})$}

This metric allows to measure the continuity of the receiving flow at the collector (a highreceiving rate provides a good quality of multimedia stream). Otherwise, it is the average rate of received packets per unit of time.

$$
R R=\frac{N_{r p}}{T_{r p}-T_{1}}
$$

Nrp: Number of received packets.

Trp: Reception time of the last packet.

T1: Reception time of the first packet.

\section{Receiving rate}

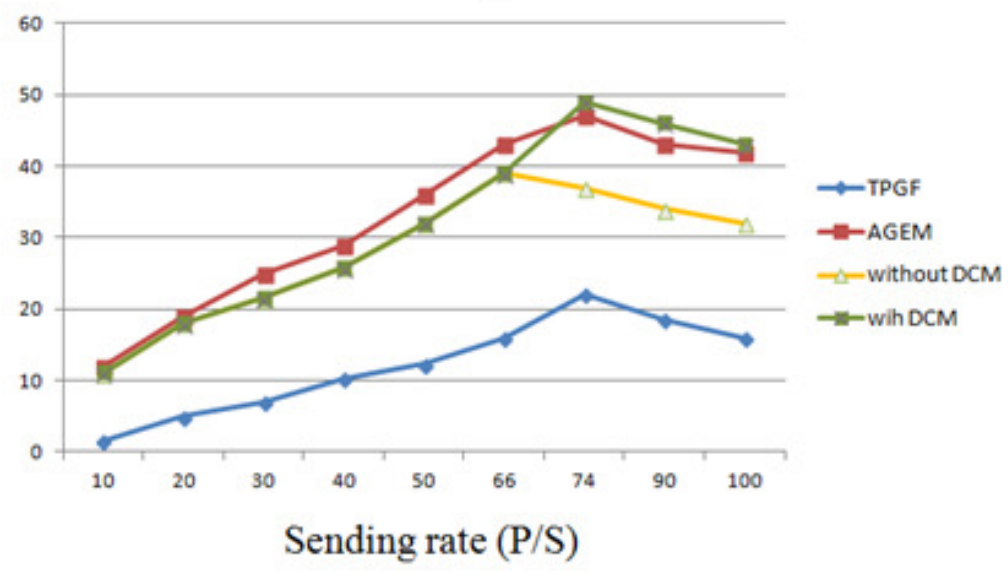

Figure 3. Impact of number of failed nodes on receiving rate curative

According to Figure.3, TPGF receiving rate is much lower, due to high rate of lost packets caused by number paths reduction, while AGEM receiving rate is lower than with DCM, due to overflow of waiting queues, when holes are detected. When the number of paths is reduced, sensor nodes 
are more loaded and queues are faster overflowed and a more important packet loss is recorded. Simulations results shows that using DCM takes advantage by keeping higher the number of paths, which consequently improves the receiving rate.

\section{CONCLUSION}

In this work we presented a dynamic curative mechanism of fault tolerance for geographic routing dedicated to multimedia streams and faulty wireless sensor networks, which tolerate failures and adapts to topology changes (dynamic holes and node's mobility). Simulation results show that our solution provides better performance and satisfies QoS requirements for multimedia transmission especially in terms of delivery rate and receiving rate.

More importantly, our dynamic curative mechanism of fault tolerance, can be adapted to any geographic routing protocol, which aims to improve the reliability of transmitted data especially in case of communication links break.

\section{REFERENCES}

[1] Sun, H. Luo, and S. K. Das. A trust-based framework for faulttolerant data aggregation in wireless multimedia sensor networks. IEEE Transactions on Dependable and Secure Computing, 9(6):785797, Nov 2012.

[2] A. Ouadjaout, Y. Challal, N. Lasla, and M. Bagaa. Seif: Secure and efficient intrusion-fault tolerant routing protocol for wireless sensor networks. In Availability, Reliability and Security, 2008. ARES 08. Third International Conference on, pages 503-508, March 2008.

[3] S. Dave and A. Raghuvanshi. Fault tolerance techniques in distributed system. International Journal of Engineering Innovation and Research, 1(2):124-130, March 2012.

[4] K. Wang, L. Wang, C. Ma, L. Shu, and J. Rodrigues. Geographic routing in random duty-cycled wireless multimedia sensor networks. In 2010 IEEE Globecom Workshops, pages 230-234, Dec 2010.

[5] L. Shu, Y. Zhang, L. T. Yang, Y. Wang, M. Hauswirth, and N. Xiong. Tpgf: geographic routing in wireless multimedia sensor networks. Telecommunication Systems, 44(1):79-95, 2010.

[6] R. E. Ahmed. A fault-tolerant, energy-efficient routing protocol for wireless sensor networks. In Information and Communication Technology Research (ICTRC), 2015 International Conference on, pages 175-178, May 2015.

[7] D. B. Johnson and D. A. Maltz. Dynamic source routing in ad hoc wireless networks. In Mobile computing, pages 153-181. Springer, 1996.

[8] C. Kavitha and K. V. Viswanatha. An energy efficient fault tolerant multipath (eeftm) routing protocol for wireless sensor networks. In Advance Computing Conference, 2009. IACC 2009. IEEE International, pages 746-751, March 2009.

[9] Z. Hamid, F. B. Hussain, and J. Y. Pyun. Delay and link utilization aware routing protocol for wireless multimedia sensor networks. Multimedia Tools and Applications, pages 1-22, 2015.

[10] N. Ahmed, S. S. Kanhere, and S. Jha. A pragmatic approach to area coverage in hybrid wireless sensor networks. Wireless Communications and Mobile Computing, pages 23-45, 2011.

[11] A. Ahmed, K. A. Bakar, M. I. Channa, K. Haseeb, and A. W. Khan. Terp: A trust and energy aware routing protocol for wireless sensor network. IEEE Sensors Journal, 15(12):6962-6972, Dec 2015. 
[12] S. Ozdemir. Functional reputation based data aggregation for wireless sensor networks. In 2008 IEEE International Conference on Wireless and Mobile Computing, Networking and Communications, pages 592-597, Oct 2008.

[13] G. Han, J. Jiang, L. Shu, J. Niu., and H. C. Chao. Management and applications of trust in wireless sensor networks: A survey. J. Comput. Syst. Sci., 80(3):602-617, 2014.

[14] M. N. Bouatit, S. Boumerdassi, and A. Djama. A Geographic Multipath Routing Protocol for Wireless Multimedia Sensor Network. In International Conference on Mobile, Secure and Programmable Networking, pages 99-108, June 2016.

[15] P. Levis and D. Gay. TinyOS Programming. Cambridge University Press, New York, NY, USA, 1st edition, 2009.

[16] S. Medjiah, T. Ahmed, and F. Krief. Agem: Adaptive greedy-compass energy-aware multipath routing protocol for wmsns. In 2010 7th IEEE Consumer Communications and Networking Conference, pages 1-6, Jan 2010.

\section{AUTHORS}

Mohamed Nacer Bouatit is currently working toward the $\mathrm{PhD}$ degree in computer science at CEDRIC-CNAM laboratory in Paris. He received a Master in web technologies from Telecom Bretagne School in 2011 and the engineer degree in computer science from the Polytechnic School of Algiers (EMP) in 2006. His research interests include wireless sensor networks, multimedia transmission, fault tolerance and energy efficiency.

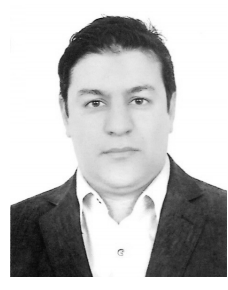

Selma Boumerdassi is an Associate Professor at Conservatoire National des Arts et Métiers, Paris. She received a PhD in Computer Science from University of Versailles in 1998, where she also served as an Assistant Professor from 1998 to 2000. Her research interests include wireless and mobile networks, with a special focus on the impact and use of social networks. She worked on several national projects and served as an expert for the evaluation of French national projects (ANR). She is the author of more than 50 articles and serves as a TPC member for various international journals and conferences.

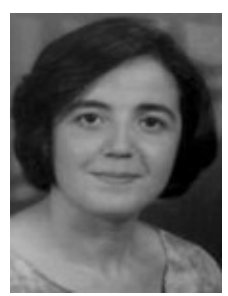

